\title{
THE MAXIMUM OF A SYMMETRIC NEXT NEIGHBOR WALK ON THE NONNEGATIVE INTEGERS
}

\author{
ORA E. PERCUS *** AND \\ JEROME K. PERCUS, ${ }^{*}$ New York University
}

\begin{abstract}
We consider a one-dimensional discrete symmetric random walk with a reflecting boundary at the origin. Generating functions are found for the two-dimensional probability distribution $\mathbb{P}\left\{S_{n}=x, \max _{1 \leq j \leq n} S_{n}=a\right\}$ of being at position $x$ after $n$ steps, while the maximal location that the walker has achieved during these $n$ steps is $a$. We also obtain the familiar (marginal) one-dimensional distribution for $S_{n}=x$, but more importantly that for $\max _{1 \leq j \leq n} S_{j}=a$ asymptotically at fixed $a^{2} / n$. We are able to compute and compare the expectations and variances of the two one-dimensional distributions, finding that they have qualitatively similar forms, but differ quantitatively in the anticipated fashion.
\end{abstract}

Keywords: One-dimensional random walk; statistics of the maximum; discrete probability; asymptotic techniques

2010 Mathematics Subject Classification: Primary 60G50

Secondary $60 \mathrm{~J} 10$

\section{Introduction}

Non-Markovian chains constitute a field of increasing activity. A dominant philosophical motif is that of a hidden Markovian chain [1], a marginal process on a higher dimensional state space. The analysis of sequences in biopolymers [7, pp. 67-69] as hidden Markov chains is a primitive version with a small underlying state space.

We were led to consider the problem analyzed in this paper during a study of reinforced random walks, next neighbor on a one-dimensional half lattice. The aim of this paper is to find the distribution of $A_{n} \equiv \max _{1 \leq i \leq n} S_{i}$, where $S_{i}$ is the location of the walker after $i$ steps. There are numerous ways to solve this problem, but we intentionally want to choose one that is extendable to a class of reinforced random walks, namely the hidden Markov viewpoint mentioned above. Before doing so, however, it is worth asking what sort of qualitative behavior to expect. Of course, we will have, asymptotically in $n, \mathbb{E}\left(S_{n}\right) \propto n^{1 / 2}$, but the maximum sojourn after $n$ steps must exceed or equal $S_{n}$. How much more? But $\left\{A_{i}\right\}$ rectifies the fluctuation in $\left\{S_{i}\right\}$; hence, $A_{n}$ might be expected to have a variance, highly reduced from that of $S_{n}$. How much less? The limited objective of this paper is to answer these questions by first computing the two-dimensional distribution of $\left(S_{n}, A_{n}\right)$ as $n$ varies and then the distribution of the random variable $A_{n}$.

Received 27 February 2013; revision received 9 April 2013.

* Postal address: Courant Institute, New York University, 251 Mercer Street, New York, NY 10012, USA.

** Email address: percus@cims.nyu.edu 


\section{Distribution and moments of $S_{n}$}

The basic system that we analyze is that of a random walk on the integer lattice $x \geq 0$. The jump $X_{i}$ at the $i$ th step is next neighbor

$$
X_{i}= \pm 1
$$

the walker starts at the origin, so that its location after $n$ steps is

$$
S_{n}=\sum_{i=1}^{n} X_{i} .
$$

Let us first review the properties of the distribution function

$$
P_{n}(x)=\mathbb{P}\left\{S_{n}=x\right\}
$$

We confine our attention to a symmetric walk reflected at the origin, so that

$$
\mathbb{P}\left\{X_{i}= \pm 1 \mid S_{i-1} \neq 0\right\}=\frac{1}{2}, \quad \mathbb{P}\left\{X_{i}=1 \mid S_{i-1}=0\right\}=1 .
$$

The first jump must be from $x=0$ to $x=1$, and so we can take the following equation as our initial condition:

$$
P_{1}(x)=\delta_{x, 1} .
$$

The analysis of (2.1) under (2.2) and (2.3) is routine. We have

$$
\begin{gathered}
\mathbb{P}\left\{S_{1}=x\right\}=\delta_{x, 1}, \\
\mathbb{P}\left\{S_{n}=0\right\}=\frac{1}{2} \mathbb{P}\left\{S_{n-1}=1\right\} \quad \text { for } n \geq 2, \\
\mathbb{P}\left\{S_{n}=1\right\}=\frac{1}{2} \mathbb{P}\left\{S_{n-1}=2\right\}+\mathbb{P}\left\{S_{n-1}=0\right\}, \\
\mathbb{P}\left\{S_{n}=x\right\}=\frac{1}{2} \mathbb{P}\left\{S_{n-1}=x+1\right\}+\frac{1}{2} \mathbb{P}\left\{S_{n-1}=x-1\right\} \quad \text { for } x \geq 2,
\end{gathered}
$$

which is readily solved (index and argument must have the same parity) as

$$
\begin{gathered}
P_{2 n}(0)=\frac{1}{2^{2 n}}\left(\begin{array}{c}
2 n \\
n
\end{array}\right), \\
P_{2 n}(2 x)=\frac{2}{2^{2 n}}\left(\begin{array}{c}
2 n \\
n-x
\end{array}\right) \text { for } x>0, \\
P_{2 n+1}(2 x+1)=\frac{1}{2^{2 n}}\left(\begin{array}{c}
2 n+1 \\
n-x
\end{array}\right) \text { for } x \geq 0 .
\end{gathered}
$$

Observe that (2.4) can also be obtained directly from a nonreflecting walk from the origin to $\pm x$ - a trivial combinatorial problem - by reflecting all subwalks on the negative axis to the positive axis. This is because the probability of a walker arriving at the origin, then jumping to \pm 1 , is 1 , as in the reflecting case.

Our definition of reflection does not correspond to that of Feller [3, p. 436], and Takács [9, p. 19], where the walker is not allowed to pass a boundary at $x=\frac{1}{2}$. Instead, when the walker is at $x=1$, the next step takes it to $x=2$ with probability $\frac{1}{2}$ or it stays at $x=1$ with probability $\frac{1}{2}$. However, Kac [5] and Percus [8] treated this walk as a Markov chain with $2 \times 2$ transition matrix, equivalent to what we do here. 
Mean and variance are the leading properties of a random walk, and by direct summation we readily find that

$$
\mathbb{E}\left(S_{2 n}\right)=\frac{2 n}{2^{2 n}}\left(\begin{array}{c}
2 n \\
n
\end{array}\right), \quad \operatorname{var}\left(S_{2 n}\right)=2 n\left(1-2 n\left(\left(\begin{array}{c}
2 n \\
n
\end{array}\right) / 2^{2 n}\right)^{2}\right),
$$

with a similar result for $S_{2 n+1}$. In both cases, use of the Stirling approximation shows directly that

$$
\lim _{n \rightarrow \infty} \frac{\mathbb{E}\left(S_{n}\right)}{n^{1 / 2}}=\sqrt{\frac{2}{\pi}}, \quad \lim _{n \rightarrow \infty} \frac{\operatorname{var}\left(S_{n}\right)}{n}=1-\frac{2}{\pi} \sim 0.36,
$$

establishing a standard against which other properties of the walk can be compared-the main objective of this paper.

\section{The joint distribution $\mathbb{P}\left\{S_{n}=x, A_{n}=a\right\}$} by

Consider a random walk on the integer lattice $x \geq 0, a \geq 1$, with joint distribution defined

$$
P_{n}(x, a) \equiv \mathbb{P}\left\{S_{n}=x, A_{n}=a\right\}, \quad \text { where } S_{k}=\sum_{i=1}^{k} X_{i}, X_{i}= \pm 1 .
$$

As in (2.2) we deal with a symmetric random walk reflected at the origin, and the walk starts at the origin, i.e.

$$
P_{1}(x, a)=\delta_{x, 1} \delta_{a, 1} .
$$

Since $a$ has not changed from its prior value when $x<a$, we have

$$
\begin{gathered}
P_{n+1}(x, a)=\frac{1}{2} P_{n}(x-1, a)+\frac{1}{2} P_{n}(x+1, a) \quad \text { for } 1<x<a, \\
P_{n+1}(1, a)=P_{n}(0, a)+\frac{1}{2} P_{n}(2, a)\left(1-\delta_{a, 1}\right), \\
P_{n+1}(0, a)=\frac{1}{2} P_{n}(1, a) .
\end{gathered}
$$

But $a$ increases from its prior value with probability $\frac{1}{2}$ when $x=a \geq 1$, and, for $a \geq 2$,

$$
P_{n+1}(a, a)=\frac{1}{2} P_{n}(a-1, a-1)+\frac{1}{2} P_{n}(a-1, a) .
$$

We can now combine (3.1), (3.2), (3.3), and (3.4) on the space defined by

$$
0 \leq x \leq a, \quad a \geq 2,
$$

obtaining, for $n \geq 1$,

$$
\begin{aligned}
P_{n+1}(x, a)= & \frac{1}{2}\left(1+\delta_{x, 1}-\delta_{x, a+1}\right) P_{n}(x-1, a) \\
& +\frac{1}{2} P_{n}(x+1, a)+\frac{1}{2} \delta_{x, a} P_{n}(a-1, a-1)\left(1-\delta_{a, 1}\right)
\end{aligned}
$$

and initial condition (3.2). Note that the condition $P_{n}(x, a)=0$ for $x>a$, satisfied initially, is automatically satisfied under iteration of (3.5).

Our task now is to solve (3.5), which we do in standard fashion by first introducing the generating function, convergent for $|\lambda|<1$,

$$
P(\lambda, x, a)=\sum_{n=1}^{\infty} \lambda^{n} P_{n}(x, a)=\lambda P_{1}(x, a)+\sum_{n=1}^{\infty} \lambda^{n+1} P_{n+1}(x, a) .
$$


It follows at once from (3.5) that

$$
\begin{aligned}
P(\lambda, x, a)= & \lambda P_{1}(x, a)+\frac{\lambda}{2}\left(1+\delta_{x, 1}-\delta_{x, a+1}\right) P(\lambda, x-1, a) \\
& +\frac{\lambda}{2} P(\lambda, x+1, a)+\frac{\lambda}{2} \delta_{x, a} P(\lambda, a-1, a-1)\left(1-\delta_{a, 1}\right) .
\end{aligned}
$$

Further simplification is then achieved by going over to the double generating function

$$
\tilde{P}(\lambda, u, a) \equiv \sum_{n=1}^{\infty} \sum_{x=0}^{\infty} \lambda^{n} u^{x} P_{n}(x, a)=\sum_{x=0}^{a} P(\lambda, x, a) u^{x},
$$

where we have used the fact that $P_{n}(x, a)=0$ for $x>a$, and this also establishes that $\tilde{P}(\lambda, u, a)$ is a polynomial in $u$ of degree $a$, thereby convergent for all $u$. Summing (3.6) over $x$, with weight $u^{x}$, we find after some minor algebra that

$$
\begin{aligned}
\left(u^{2}-\frac{2 u}{\lambda}+1\right) \tilde{P}(\lambda, u, a)= & -2 u^{2} \delta_{a, 1}+\left(1-u^{2}\right) P(\lambda, 0, a)+u^{a+2} P(\lambda, a, a) \\
& -u^{a+1} P(\lambda, a-1, a-1)\left(1-\delta_{a, 1}\right) .
\end{aligned}
$$

Solving (3.7) is fairly straightforward. First, take the special case $a=1$, i.e.

$$
\left(u^{2}-\frac{2 u}{\lambda}+1\right) \tilde{P}(\lambda, u, 1)=-2 u^{2}+\left(1-u^{2}\right) P(\lambda, 0,1)+u^{3} P(\lambda, 1,1)
$$

and introduce the zeroes of $u^{2}-2 u / \lambda+1=0$, i.e.

$$
u_{1}=\theta=\frac{1-\sqrt{1-\lambda^{2}}}{\lambda}, \quad u_{2}=\frac{1}{\theta}=\frac{1+\sqrt{1-\lambda^{2}}}{\lambda} .
$$

Taking $u=\theta$, and then $u=1 / \theta$ in (3.8), we have

$$
\begin{aligned}
& 0=-2 \theta^{2}+\left(1-\theta^{2}\right) P(\lambda, 0,1)+\theta^{3} P(\lambda, 1,1), \\
& 0=-2 \theta^{-2}+\left(1-\theta^{-2}\right) P(\lambda, 0,1)+\theta^{-3} P(\lambda, 1,1),
\end{aligned}
$$

and, on eliminating $P(\lambda, 0,1)$,

$$
P(\lambda, 1,1)=\frac{2\left(\theta+\theta^{-1}\right)}{\theta^{2}+\theta^{-2}}=\frac{\lambda}{1-\lambda^{2} / 2} .
$$

The case $a>1$ can be treated the same way. Using (3.7), we obtain

$$
\begin{aligned}
& 0=\left(1-\theta^{2}\right) P(\lambda, 0, a)+\theta^{a+2} P(\lambda, a, a)-\theta^{a+1} P(\lambda, a-1, a-1), \\
& 0=\left(1-\theta^{-2}\right) P(\lambda, 0, a)+\theta^{-a-2} P(\lambda, a, a)-\theta^{-a-1} P(\lambda, a-1, a-1),
\end{aligned}
$$

and, eliminating $P(\lambda, 0, a)$,

$$
P(\lambda, a, a)=\frac{\theta^{a}+\theta^{-a}}{\theta^{a+1}+\theta^{-(a+1)}} P(\lambda, a-1, a-1) .
$$


Starting with (3.9) and iteratively applying (3.10), we conclude that

$$
P(\lambda, a, a)=2 \frac{\theta+\theta^{-1}}{\theta^{a+1}+\theta^{-(a+1)}}=\frac{4 / \lambda}{\theta^{a+1}+\theta^{-(a+1)}},
$$

which is also valid for $a=1$, and leading via the first equality of (3.9) and the first of (3.11) to

$$
P(\lambda, 0, a)=\frac{2 \theta^{2}}{1-\theta^{2}} \delta_{a, 1}+\frac{\theta^{a+1}}{1-\theta^{2}} P(\lambda, a-1, a-1)\left(1-\delta_{a, 1}\right)-\frac{\theta^{a+2}}{1-\theta^{2}} P(\lambda, a, a)
$$

(note the convention that $P(\lambda, 0,0)=0$ ). The net effect, substituting back into (3.7), is that

$$
\begin{aligned}
\tilde{P}(\lambda, u, a) & \left(u^{2}-\frac{2 u}{\lambda}+1\right) \\
= & \left(\left(1-u^{2}\right) \frac{\theta^{a+1}}{1-\theta^{2}}-u^{a+1}\right)\left(P(\lambda, a-1, a-1)\left(1-\delta_{a, 1}\right)+2 \delta_{a, 1}\right) \\
& -\left(\left(1-u^{2}\right) \frac{\theta^{a+2}}{1-\theta^{2}}-u^{a+2}\right) P(\lambda, a, a)
\end{aligned}
$$

or

$$
\begin{aligned}
(u-\theta)\left(u-\frac{1}{\theta}\right) \tilde{P}(\lambda, u, a)= & \left(\left(1-u^{2}\right) \frac{\theta^{a+1}}{1-\theta^{2}}-u^{a+1}\right) 2 \frac{\theta+\theta^{-1}}{\theta^{a}+\theta^{-a}} \\
& -\left(\left(1-u^{2}\right) \frac{\theta^{a+2}}{1-\theta^{2}}-u^{a+2}\right) 2 \frac{\theta+\theta^{-1}}{\theta^{a+1}+\theta^{-(a+1)}} .
\end{aligned}
$$

Note that from (3.12) we conclude

$$
\mathbb{P}\left\{A_{n}=a\right\}=\text { the coefficient of } \lambda^{n} \text { in } \frac{1}{1-\lambda}\left(\frac{2}{\theta^{a}+\theta^{-a}}-\frac{2}{\theta^{a+1}+\theta^{-(a+1)}}\right)
$$

and, since

$$
\mathbb{P}\left\{A_{n-1}=a-1\right\}=\text { the coefficient of } \lambda^{n-1} \text { in } \frac{1}{1-\lambda}\left(\frac{2}{\theta^{a-1}+\theta^{-(a-1)}}-\frac{2}{\theta^{a}+\theta^{-a}}\right),
$$

we also find that

$$
\begin{aligned}
\mathbb{P}\left\{A_{n}=\right. & a \text { for the first time in the } n \text {th step }\} \\
= & \text { the coefficient of } \lambda^{n} \text { in } \frac{1}{1-\lambda}\left(\frac{2}{\theta^{a}+\theta^{-a}}-\frac{2}{\theta^{a+1}+\theta^{-(a+1)}}\right) \\
& - \text { the coefficient of } \lambda^{n-1} \text { in } \frac{1}{1-\lambda}\left(\frac{2}{\theta^{a-1}+\theta^{-(a-1)}}-\frac{2}{\theta^{a}+\theta^{-a}}\right)
\end{aligned}
$$

(see (4.1)-(4.3) for details).

\section{The limiting moments of $A_{n}$}

Our objective is to examine the characteristics of the non-Markovian random variable $A_{n} \equiv \max _{1 \leq j \leq n} S_{n}$, which of course corresponds to obtaining the marginal distribution in 
which $\mathbb{P}\left\{S_{n}=x, \max _{1 \leq i \leq n} S_{i}=a\right\}$ is summed over $x$. The complementary marginal, summed over $a$, is just the usual Markovian walk of $\mathbb{P}\left\{S_{n}=x\right\}$, whose solution was given in Section 2 .

We have seen in (3.12) that

$$
\begin{aligned}
\left(u^{2}-\frac{2 u}{\lambda}+1\right) \tilde{P}(\lambda, u, a)= & \left(\left(1-u^{2}\right) \frac{\theta^{a+1}}{1-\theta^{2}}-u^{a+1}\right) 2 \frac{\theta+\theta^{-1}}{\theta^{a}+\theta^{-a}} \\
& -\left(\left(1-u^{2}\right) \frac{\theta^{a+2}}{1-\theta^{2}}-u^{a+2}\right) 2 \frac{\theta+\theta^{-1}}{\theta^{a+1}+\theta^{-(a+1)}} .
\end{aligned}
$$

The generating function for the marginal distribution of $A_{n}$ is then found by summing over $S_{n}=x$, equivalent to setting $u=1$ in (4.1), i.e.

$$
\left(1-\frac{1}{\lambda}\right) \tilde{P}(\lambda, 1, a)=\frac{\theta+\theta^{-1}}{\theta^{a+1}+\theta^{-(a+1)}}-\frac{\theta+\theta^{-1}}{\theta^{a}+\theta^{-a}}
$$

or, since $\theta+\theta^{-1}=2 / \lambda$,

$$
(1-\lambda) \tilde{P}(\lambda, 1, a)=\frac{2}{\theta^{a}+\theta^{-a}}-\frac{2}{\theta^{a+1}+\theta^{-(a+1)}} .
$$

It is then simple to construct the double generating function

$$
\begin{aligned}
Q(\lambda, z) & \equiv \sum_{1,1}^{\infty, \infty} \lambda^{n} z^{a} \mathbb{P}\left\{A_{n}=a\right\} \\
& =\sum_{1}^{\infty} z^{a} \tilde{P}(\lambda, 1, a) \\
& =\frac{1}{1-\lambda} \sum_{1}^{\infty} z^{a}\left(\frac{2}{\theta^{a}+\theta^{-a}}-\frac{2}{\theta^{a+1} \theta^{-(a+1)}}\right) \\
& =\frac{1}{1-\lambda}\left(\sum_{1}^{\infty}\left(z^{a}-z^{a-1}\right) \frac{2}{\theta^{a}+\theta^{-a}}+\lambda\right) \\
& =\frac{\lambda}{1-\lambda}-\frac{1-z}{1-\lambda} \sum_{1}^{\infty} z^{a} \frac{2}{\theta^{a}+\theta^{-a}} .
\end{aligned}
$$

The factorial moments of $\left\{A_{n}\right\}$ are of course obtained by $z$-differentiations of $Q(\lambda, z)$ at $z=1$, or directly in the fashion of (4.4) (using the familiar recurrence relation of binomial coefficients), i.e. for $k \geq 1$,

$$
\sum_{n} \lambda^{n} \mathbb{E}\left(\begin{array}{c}
A_{n} \\
k
\end{array}\right)=\frac{1}{1-\lambda} \sum_{a=1}^{\infty}\left(\begin{array}{c}
a \\
k-1
\end{array}\right) \frac{2}{\theta^{a}+\theta^{-a}} .
$$

Our objective is to obtain the asymptotic form of $\mathbb{E}\left(\begin{array}{c}A_{n} \\ k\end{array}\right)$ as $n \rightarrow \infty$. Our claim is that this has the same form as the $k$ th moment of $S_{n}, \mathbb{E}\left(S_{n}^{k}\right)$, which we know is proportional to $n^{k / 2}$. In other words, we want to find the constant $C_{k}$ in the postulated relation

$$
\lim _{n \rightarrow \infty} \mathbb{E}\left(\begin{array}{c}
A_{n} \\
k
\end{array}\right) / n^{k / 2}=C_{k}
$$


It is not obvious that this limit exists because $\left(1 / n^{k / 2}\right) \mathbb{E}\left(\begin{array}{c}A_{n} \\ k\end{array}\right)$ may have persistent oscillations when $n \rightarrow \infty$. Therefore, we will instead use a generalized limit in the sense of Abel or Cesaro, see e.g. [6, p. 583], in which a suitable running average is performed before the limit is taken. Prototypical is one form of the Abel limit theorem which states that if

$$
\lim _{n \rightarrow \infty} a_{n}=A \text { then } \lim _{\lambda \rightarrow 1-}(1-\lambda) \sum_{n=1}^{\infty} a_{n} \lambda^{n}=A .
$$

This is readily proved by decomposing the sum into two sums, i.e.

$$
(1-\lambda) \sum_{n=1}^{\infty} a_{n} \lambda^{n}=(1-\lambda) \sum_{n=1}^{(1-\lambda)^{-1 / 2}-1} a_{n} \lambda^{n}+(1-\lambda) \sum_{n=(1-\lambda)^{-1 / 2}}^{\infty} a_{n} \lambda^{n},
$$

and observing that the first term tends to 0 while the second term tends to $A$ as $\lambda \rightarrow 1-$. Equation (4.7) can be generalized using the same decomposition of the sum (as in (4.8)) to read if

$$
\lim _{n \rightarrow \infty}\left(1 /\left(\begin{array}{l}
n \\
p
\end{array}\right)\right) a_{n}=C \text { then } \lim _{\lambda \rightarrow 1-}(1-\lambda)^{1+p} \sum_{n=1}^{\infty} \lambda^{n-p} a_{n}=C .
$$

Since $\left(\begin{array}{l}n \\ p\end{array}\right) / n^{p}$ tends to $1 / p$ ! as $n \rightarrow \infty$, we therefore define

$$
\lim _{n \rightarrow \infty}^{*} n^{-p} a_{n} \equiv \lim _{\lambda \rightarrow 1-}(1-\lambda)^{1+p} \sum_{n=1}^{\infty} \frac{1}{p !} \lambda^{n-p} a_{n} .
$$

If $\lim _{n \rightarrow \infty} n^{-p} a_{n}$ exists then $\lim _{n \rightarrow \infty}^{*} a_{n} n^{-p}$ has the same value. However, lim* may exist even when lim does not.

We now apply (4.9) to (4.5) to obtain the lim* version of (4.6) as follows:

$$
\begin{aligned}
\lim _{n \rightarrow \infty}^{*} \frac{1}{n^{k / 2}} \mathbb{E}\left(\begin{array}{c}
A_{n} \\
k
\end{array}\right) & =\lim _{\lambda \rightarrow 1-}(1-\lambda)^{1+k / 2} \sum_{n=1}^{\infty} \frac{1}{(k / 2) !} \lambda^{n-k / 2} \mathbb{E}\left(\begin{array}{c}
A_{n} \\
k
\end{array}\right) \\
& =\lim _{\lambda \rightarrow 1-}\left(\frac{1-\lambda}{\lambda}\right)^{k / 2} \sum_{a=1}\left(\begin{array}{c}
a \\
k-1
\end{array}\right) \frac{2}{\theta^{a}+\theta^{-a}} \frac{1}{(k / 2) !} .
\end{aligned}
$$

Set $\theta=\mathrm{e}^{-t}$; therefore,

$$
\frac{2}{\theta^{a}+\theta^{-a}}=\frac{1}{\cosh a t}, \quad \frac{1-\lambda}{\lambda}=\cosh t-1,
$$

and (4.10) can be written as

$$
\lim _{n \rightarrow \infty}^{*} \frac{1}{n^{k / 2}} \mathbb{E}\left(\begin{array}{c}
A_{n} \\
k
\end{array}\right)=\lim _{t \rightarrow 0+}(\cosh t-1)^{k / 2} \frac{1}{(k / 2) !} \sum_{a=1}\left(\begin{array}{c}
a \\
k-1
\end{array}\right) \frac{1}{\cosh a t} .
$$

Since $(\cosh t-1)^{k / 2} /\left(t^{2} / 2\right)^{k / 2} \rightarrow 1$ as $t \rightarrow 0$ for $k \geq 1$, (4.11) becomes

$$
\begin{aligned}
\lim _{n \rightarrow \infty}^{*} \frac{1}{n^{k / 2}} \mathbb{E}\left(\begin{array}{c}
A_{n} \\
k
\end{array}\right) & =\lim _{t \rightarrow 0+} \frac{t^{k}}{2^{k / 2}(k / 2) !} \sum_{a=1}\left(\begin{array}{c}
a \\
k-1
\end{array}\right) \frac{1}{\cosh a t} \\
& =\lim _{t \rightarrow 0+}\left(\frac{t}{\sqrt{2}}\right)^{k} \frac{1}{(k / 2) !} \sum_{a=k-1}\left(\begin{array}{c}
a \\
k-1
\end{array}\right) \frac{1}{\cosh a t} .
\end{aligned}
$$


But,

$$
\begin{aligned}
\lim _{t \rightarrow 0}\left(\frac{t}{\sqrt{2}}\right)^{k} \frac{1}{(k / 2) !} \sum_{a=k-1}\left(\begin{array}{c}
a \\
k-1
\end{array}\right) \frac{1}{\cosh a t} \\
\quad=\lim _{t \rightarrow 0} \frac{t^{k} 2^{-k / 2}}{(k / 2) !}\left(\sum_{a=k-1}^{\alpha / t-1}+\sum_{a=\alpha / t}^{\beta / t-1}+\sum_{a=\beta / t}^{\infty}\right)\left(\begin{array}{c}
a \\
k-1
\end{array}\right) \frac{1}{\cosh a t}
\end{aligned}
$$

It can be verified that the contribution of the third sum is a function of $\beta$ and $t$ which tends to 0 as $\beta \rightarrow \infty$ for any $t$, and the contribution of the first sum tends to 0 as $\alpha \rightarrow 0$ for any $t$. Now, the contribution of the second sum involves a Riemann sum which converges to a Riemann integral, i.e.

$$
\frac{1}{2^{k / 2}(k / 2) !(k-1) !} \int_{\alpha}^{\beta} \frac{b^{k-1}}{\cosh b} \mathrm{~d} b \rightarrow \frac{1}{2^{k / 2}(k / 2) !(k-1) !} \int_{0}^{\infty} \frac{b^{k-1}}{\cosh b} \mathrm{~d} b
$$

as $\alpha \rightarrow 0$ and $\beta \rightarrow \infty$. From (4.12) and (4.13) we conclude that

$$
\lim _{n \rightarrow \infty}^{*} n^{-k / 2} \mathbb{E}\left(\begin{array}{c}
A_{n} \\
k
\end{array}\right)=\frac{1}{2^{k / 2}(k / 2) !(k-1) !} \int_{0}^{\infty} \frac{b^{k-1}}{\cosh b} \mathrm{~d} b
$$

or, equally well for $k \geq 1$,

$$
\lim _{n \rightarrow \infty}^{*} n^{-k / 2} \mathbb{E}\left(A_{n}^{k}\right)=\frac{k}{2^{k / 2}(k / 2) !} \int_{0}^{\infty} \frac{b^{k-1}}{\cosh b} \mathrm{~d} b .
$$

The most important examples will be (see [2] for evaluation of the integrals)

$$
\begin{aligned}
& \lim _{n \rightarrow \infty}^{*} n^{-1 / 2} \mathbb{E}\left(A_{n}\right)=\frac{1}{\sqrt{2} \frac{1}{2} \sqrt{\pi}} \int_{0}^{\infty} \frac{\mathrm{d} b}{\cosh b}=\sqrt{\frac{\pi}{2}} \quad(k=1), \\
& \lim _{n \rightarrow \infty} * \frac{1}{n} \mathbb{E}\left(A_{n}^{2}\right)=\int_{0}^{\infty} \frac{b \mathrm{~d} b}{\cosh b}=2 G=1.83193 \quad(k=2),
\end{aligned}
$$

where $G$ is the Catalan constant (see [4]), from which we obtain

$$
\lim _{n \rightarrow \infty} * \frac{1}{n} \operatorname{var}\left(A_{n}\right)=0.26113 \ldots
$$

Including the results of (2.5), we have

$$
\begin{array}{rlrl}
\lim \frac{1}{\sqrt{n}} \mathbb{E}\left(S_{n}\right) & \equiv \sqrt{\frac{2}{\pi}}=0.7979, & \lim ^{*} \frac{1}{\sqrt{n}} \mathbb{E}\left(A_{n}\right)=\sqrt{\frac{\pi}{2}}=1.2533, \\
\lim \frac{1}{\sqrt{n}} \operatorname{var}\left(S_{n}\right)=0.36, & \lim ^{*} \frac{1}{n} \operatorname{var}\left(A_{n}\right)=0.26113 .
\end{array}
$$

From the definitions of $A_{n}$ and $S_{n}$, the mean of $A_{n}$ might have been much larger than the mean of $S_{n}$; it is not. Also, the variance of $A_{n}$ might have been much smaller than the variance of $S_{n}$; it is not. 
A heuristic version of the process used in obtaining (4.14) can be carried out for (4.4), resulting in

$$
\begin{aligned}
Q(\lambda, z)= & \frac{1}{1-\lambda}\left(\lambda+\frac{1}{2}(1-z)\right) \\
& -\frac{1-z}{1-\lambda} \frac{1}{2 \operatorname{sech}^{-1} \lambda}\left(\left(\psi\left(\frac{1}{4} \frac{\log z}{\operatorname{sech}^{-1} \lambda}\right)+\frac{3}{4}\right)-\psi\left(\frac{1}{4} \frac{\log z}{\operatorname{sech}^{-1} \lambda}+\frac{1}{4}\right)\right),
\end{aligned}
$$

where $\psi$ is the dilogarithm function.

\section{The limiting distribution of $A_{n}$}

The moments supply crucial information as to the nature of the distribution of the random variation $A_{n}$. But can we find this distribution, i.e.

$$
Q_{n}(a) \equiv \mathbb{P}\left\{A_{n}=a\right\},
$$

in an explicit—and usable-form? We have seen, and used, the generating function relation (4.3) with the consequence that

$$
Q_{n}(a)-Q_{n-1}(a)=\text { the coefficient of } \lambda^{n} \text { in } \frac{2}{\theta^{a}+\theta^{-a}}-\frac{2}{\theta^{a+1}+\theta^{-(a+1)}} .
$$

Our first task will be to find the coefficient of $\lambda^{n}$ in $1 /\left(\theta^{a}+\theta^{-a}\right)$. It is easy to see that

$$
\frac{2}{\theta^{a}+\theta^{-a}}=\frac{1}{T_{a}(1 / \lambda)}
$$

where $T_{a}$ is the $a$ th Chebyshev polynomial, but this is not very helpful. However, a simple partial fraction decomposition is completely effective. We have

$$
\begin{aligned}
\frac{1}{\theta^{-a}+\theta^{a}} & =\frac{\theta^{a}}{\theta^{2 a}+1} \\
& =\sum_{j=1}^{2 a} \frac{\theta_{j}^{a}}{2 a \theta_{j}^{2 a-1}}\left(\frac{1}{\theta-\theta_{j}}\right) \\
& =\frac{1}{2 a} \sum_{j=1}^{2 a} \frac{\theta_{j}^{a+1}}{\theta-\theta_{j}},
\end{aligned}
$$

where $\theta_{j}=\mathrm{e}^{(i \pi / 2 a)(2 j-1)}$. Replacing $\theta_{j}$ by $1 / \theta_{j}$ does not change the set $\left\{\theta_{j}\right\}$, and so we can replace (5.2) by its average over the two forms, i.e.

$$
\begin{aligned}
\frac{1}{\theta^{-a}+\theta^{a}} & =-\frac{1}{4 a} \sum_{1}^{2 a} \frac{\theta_{j}^{a+1}}{\theta-\theta_{j}}+\frac{\theta_{j}^{-(a+1)}}{\theta-\theta_{j}^{-1}} \\
& =\frac{1}{4 a} \sum_{1}^{2 a} \frac{\theta_{j}^{a}\left(\theta_{j}^{-1}-\theta_{j}\right)}{\theta+\theta^{-1}-\left(\theta_{j}+\theta_{j}^{-1}\right)} \\
& =\frac{i \lambda}{8 a} \sum_{1}^{2 a} \frac{(-1)^{j}\left(\theta_{j}^{-1}-\theta_{j}\right)}{1-(\lambda / 2)\left(\theta_{j}^{-1}+\theta_{j}\right)},
\end{aligned}
$$


where we have used $\theta_{j}=\exp (i \pi / 2 a)(2 j-1), \theta_{j}^{a}=-i(-1)^{j}$, and $\theta+\theta^{-1}=2 / \lambda$. It follows at once that the coefficient of $\lambda^{n}$ in $1 /\left(\theta^{a}+\theta^{-a}\right)$ is equal to

$$
\frac{1}{4 a} \sum_{0}^{2 a-1}(-1)^{j} \sin \frac{\pi}{2 a}(2 j+1) \cos ^{n-1} \frac{\pi}{2 a}(2 j+1) .
$$

Note that if we replace the summation index term $j$ in (5.3) by $2 a-1-j$, this leaves every term in (5.3) unchanged. Thus, we can replace the summation range by its lower half and multiply by 2 . Therefore, the coefficient of $\lambda^{n}$ in $1 /\left(\theta^{a}+\theta^{-a}\right)$ is equal to

$$
\frac{1}{2 a} \sum_{j=0}^{a-1}(-1)^{j} \sin \frac{\pi}{2 a}(2 j+1) \cos ^{n-1} \frac{\pi}{2 a}(2 j+1) .
$$

But then, if we replace the summation index term $j$ in (5.4) by $a-1-j$, this multiplies each term in (5.4) by $(-1)^{n+a}$, with the following four consequences.

(i) The coefficient of $\lambda^{n}$ in $1 /\left(\theta^{a}+\theta^{-a}\right)$ is not equal to 0 only if $a \equiv n(\bmod 2)$.

(ii) If $a \equiv n(\bmod 2)$ then $(5.4)$ can be reduced to its lower half-range (for odd $a$, the summand vanishes at both $(a-1) / 2$ and $(a+1) / 2)$, i.e. the coefficient of $\lambda^{n}$ in $1 /\left(\theta^{a}+\theta^{-a}\right)$ is equal to

$$
\frac{1}{a} \sum_{j=0}^{a / 2-1}(-1)^{j} \sin \frac{\pi}{2 a}(2 j+1) \cos ^{n-1} \frac{\pi}{2 a}(2 j+1) .
$$

(iii) The sum (5.5) is strictly alternating in sign, since $0<(\pi / 2 a)(2 j+1) \leq \pi / 2$.

(iv) It also follows from (i) that

$$
Q_{n}(a)-Q_{n-1}(a)=\delta_{a, 1} \delta_{n, 1}+2(-1)^{n+a}\left(\text { the coefficient of } \lambda^{n} \text { in } \frac{1}{\theta^{b}+\theta^{-b}}\right),
$$

where

$$
b= \begin{cases}a & \text { for } a \equiv n(\bmod 2), \\ a+1 & \text { for } a \equiv(n+1)(\bmod 2) .\end{cases}
$$

We can apply (5.6) at once to (5.1) by making use of the fact that $\lim _{a \rightarrow \infty} \mathbb{P}\left\{A_{n}=a\right\}=0$. It then follows from (5.5) on summing over $n$ that

$$
\begin{aligned}
& Q_{N}(a)=-\sum_{n=N+1}^{\infty}\left(Q_{n}(a)-Q_{n-1}(a)\right) \\
& =\left\{\begin{array}{l}
\frac{1}{a+1} \sum_{j}(-1)^{j} \frac{\cos ^{N}(\pi(2 j+1) / 2(a+1))}{\sin (\pi(2 j+1) / 2(a+1))} \\
-\frac{1}{a} \sum_{j}(-1)^{j} \frac{\cos ^{N+1}(\pi(2 j+1) / 2 a)}{\sin (\pi(2 j+1) / a)} \quad \text { if } N \equiv a(\bmod 2), \\
\frac{1}{a+1} \sum_{j}(-1)^{j} \frac{\cos ^{N+1}(\pi(2 j+1) / 2(a+1))}{\sin (\pi(2 j+1) / 2(a+1))} \\
-\frac{1}{a} \sum_{j}(-1)^{j} \frac{\cos ^{N}(\pi(2 j+1) / 2 a)}{\sin (\pi(2 j+1) / 2 a)} \quad \text { if } N \equiv(a+1)(\bmod 2),
\end{array}\right.
\end{aligned}
$$


which although rather complex has the necessary property of vanishing when $N<a$. Note that the sum over $n$ required to obtain (5.7) starts at $N+1$ or $N+2$ depending upon the relative parity of $N$ and $a$, and goes up in steps of 2 .

We will find the limit of $Q_{N}(a)$ as $a \rightarrow \infty$ and $N \rightarrow \infty$ at fixed $\gamma$, where

$$
\frac{a^{2}}{N}=\gamma \frac{\pi}{2}+O\left(\frac{1}{a}\right)
$$

Consider the case $N \equiv a(\bmod 2)$ in $(5.7)($ the case $N \equiv(a+1)(\bmod 2)$ proceeds similarly). After a certain amount of algebra, we obtain

$$
\begin{aligned}
& \lim _{\substack{a, N \rightarrow \infty \\
a^{2} / N=\gamma \pi / 2+O(1 / N)}}\left(\frac{a}{a+1} \frac{\cos ^{N}(\pi(2 j+1) / 2(a+1))}{\sin (\pi(2 j+1) / 2(a+1))}-\frac{\cos ^{N+1}(\pi(2 j+1) / 2 a)}{\sin (\pi(2 j+1) / 2 a)}\right) \\
& =\frac{(2 j+1)}{\gamma} \mathrm{e}^{-\pi(2 j+1)^{2} / 4 \gamma} .
\end{aligned}
$$

It therefore follows that, for fixed $\gamma$,

$$
\lim _{a \rightarrow \infty} a Q_{N}(a)=\sum_{j=0}^{\infty}(-1)^{j} \frac{(2 j+1)}{\gamma} \mathrm{e}^{-\pi(2 j+1)^{2} / 4 \gamma} .
$$

For $\gamma<1,(5.8)$ is an alternating series with a decreasing absolute value of the $j$ th term. The absolute ratio of the $j$ th term to the $(j-1)$ th term is given by $((2 j+1) /(2 j-1)) \mathrm{e}^{-2 \pi j / \gamma}$; hence,

$$
(1-\alpha) \frac{1}{\gamma} \mathrm{e}^{-\pi / 4 \gamma} \leq \lim _{a \rightarrow \infty} a Q_{N}(a) \leq \frac{1}{\gamma} \mathrm{e}^{-\pi / 4 \gamma},
$$

where $\alpha=3 \mathrm{e}^{-2 \pi / \gamma} \leq 0.0056$ for $\gamma \leq 1$. For $\gamma>1$ the rapid convergence of the series (5.8) quickly deteriorates as does the information supplied by the first term in the series. However, (5.8) does exist and absolutely converges for all $\gamma$; it is therefore necessary to replace (5.8) by a more rapidly convergent representation. This is supplied by a modification of the familiar Poisson resummation:

$$
\sum_{-\infty}^{\infty} g(j)=\sum_{k=-\infty}^{\infty} \tilde{g}(k), \quad \text { where } \tilde{g}(k) \equiv \int_{-\infty}^{\infty} g(x) \mathrm{e}^{2 \pi i k x} \mathrm{~d} x
$$

As a special case, define $g(j) \equiv \sin (\pi j / 2) f(j)$ so that $\sum_{j=-\infty}^{\infty} g(j)=\sum_{n=-\infty}^{\infty}(-1)^{n} f(2 n+$ $1)$. It then follows directly from (5.10) that

$$
\sum_{n=-\infty}^{\infty}(-1)^{n} f(2 n+1)=\frac{1}{2 i} \sum_{k=-\infty}^{\infty}(-1)^{k} \tilde{f}\left(\frac{1}{4}(2 k+1)\right) .
$$

As an example, we find at once that

$$
\gamma^{3 / 2} \sum_{n=-\infty}^{\infty}(-1)^{n}\left(n+\frac{1}{2}\right) \mathrm{e}^{-\pi \gamma(n+1 / 2)^{2}}=\sum_{k=-\infty}^{\infty}(-1)^{k}\left(k+\frac{1}{2}\right) \mathrm{e}^{-\pi(k+1 / 2)^{2} / \gamma} .
$$


Hence, (5.8) is equivalent to

$$
\lim a Q_{n}(a)=\sqrt{\gamma} \sum_{j=0}^{\infty}(-1)^{j}(2 j+1) \mathrm{e}^{-\pi \gamma(2 j+1)^{2} / 4} .
$$

Equation (5.11) now converges very rapidly, as did (5.8), and we similarly conclude that

$$
(1-\alpha) \sqrt{\gamma} \mathrm{e}^{-\pi \gamma / 4} \leq \lim _{a \rightarrow \infty} a Q_{n}(a) \leq \sqrt{\gamma} \mathrm{e}^{-\pi \gamma / 4}
$$

for $\alpha=3 \mathrm{e}^{-2 \pi \gamma} \leq 0.0056$ when $\gamma \geq 1$.

The general summation device we have used is not unknown in our particular case; it stems from the fact that (5.8) is recognized as a derivative of the Jacobi theta function, which under the Jacobi imaginary transformation is converted to (5.11).

\section{Concluding remarks}

We conclude (see (5.8) and (5.11)) that the asymptotic $\left(a \rightarrow \infty\right.$ at constant $\gamma=2 a^{2} / \pi n+$ $O(1 / a)$ ) value of the pointwise distribution $a Q_{n}(a)=a \mathbb{P}\left\{A_{n}=a\right\}$ of the maximum of our random walk has been found over the full range of $\gamma$. Furthermore, very simple estimates, (5.9) and (5.12), were obtained with a uniform maximum relative error of $\alpha=0.0056$. Coupled with the asymptotic $(n \rightarrow \infty)$ Abel-smoothed moments we presented in (4.14) and (4.15), a quite complete characterization of this process has become available.

\section{Acknowledgements}

We gratefully acknowledge the invaluable help of an anonymous referee in clarifying the presentation of this paper. Conversations with J. H. Spencer and S. R. S. Varadhan were also helpful.

\section{References}

[1] Baum, L. E. And Pitrie, T. (1966). Statistical inference for probabilistic functions of finite state Markov chains. Ann. Math. Statist. 37, 1554-1563.

[2] Erdélyi, A., Magnus, W., Oberhettinger, F. and Tricomi, F. G. (1954). Tables of Integral Transforms, Vol. I, McGraw-Hill, New York.

[3] Feller, W. (1968). An Introduction to Probability Theory and Its Applications, Vol. I, 3rd edn. John Wiley, New York.

[4] Gradshteyn, I. S. And Ryzhik, I. M. (1965). Tables of Integrals, Series, and Products, 4th edn. Academic Press, New York.

[5] KAC, M. (1947). Random walk and the theory of Brownian motion. Amer. Math. Monthly 54, 369-391.

[6] Malacrov, B. and Podkorytov, A. (2013). Real Analysis: Measures, Integrals and Applications, Springer, London.

[7] Percus, J. K. (2002). Mathematics of Genome Analysis. Cambridge University Press.

[8] Percus, O. E. (1985). Phase transition in one-dimensional random walk with partially reflecting boundaries. Adv. Appl. Prob. 17, 594-606.

[9] TAKács, L. (1960). Stochastic Processes: Problems and Solutions. Methuen, London. 